\title{
Diminished heart beat non-stationarities in congestive heart failure
}

\author{
Sabrina Camargo ${ }^{1,2}$, Maik Riedl $^{1}$, Celia Anteneodo ${ }^{2,3}$, Jürgen Kurths ${ }^{1,4,5}$ and Niels Wessel ${ }^{1}$ * \\ 1 Department of Physics, Humboldt-Universität zu Berlin, Berlin, Germany \\ 2 Department of Physics, PUC-Rio, Rio de Janeiro, Brazil \\ ${ }^{3}$ National Institute of Science and Technology for Complex Systems, Rio de Janeiro, Brazil \\ 4 Potsdam Institute for Climate Impact Research, Potsdam, Germany \\ ${ }^{5}$ Institute for Complex Systems and Mathematical Biology, University of Aberdeen, Aberdeen, UK
}

Edited by:

Zbigniew R. Struzik, The University

of Tokyo, Japan

Reviewed by:

Alberto Porta, University of Milan,

Italy

Danuta Makowiec, Gdansk

University, Poland

\section{${ }^{*}$ Correspondence:}

Niels Wessel, Department of

Physics, AG NLD Kardiovaskuläre

Physik, Humboldt-Universität zu

Berlin, Robert-Koch-Platz 4, Berlin

10115, Germany.

e-mail:niels.wessel@physik.

hu-berlin.de
Studies on heart rate variability (HRV) have become popular and the possibility of diagnosis based on non-invasive techniques compels us to overcome the difficulties originated on the environmental changes that can affect the signal. We perform a non-parametric segmentation which consists of locating the points where the signal can be split into stationary segments. By finding stationary segments we are able to analyze the size of these segments and evaluate how the signal changes from one segment to another, looking at the statistical moments given in each patch, for example, mean and variance. We analyze HRV data for 15 patients with congestive heart failure (CHF; 11 males, 4 females, age $56 \pm 11$ years), 18 elderly healthy subjects ( $E H ; 11$ males, 7 females, age $50 \pm 7$ years), and 15 young healthy subjects ( $\mathrm{YH} ; 11$ females, 4 males, age $31 \pm 6$ years). Our results confirm higher variance for $\mathrm{YH}$, and $\mathrm{EH}$, while $\mathrm{CHF}$ displays diminished variance with $p$-values $<0.01$, when compared to the healthy groups, presenting higher $\mathrm{HRV}$ in healthy subjects. Moreover, it is possible to distinguish between $\mathrm{YH}$ and $\mathrm{EH}$ with $p<0.05$ through the segmentation outcomes. We found high correlations between the results of segmentation and standard measures of HRV analysis and a connection to results of detrended fluctuation analysis (DFA). The segmentation applied to HRV studies detects aging and pathological conditions effects on the non-stationary behavior of the analyzed groups, promising to contribute in complexity analysis and providing risk stratification measures.

Keywords: heart rate variability, non-stationarity, segmentation

\section{INTRODUCTION}

It has been a long time since studies on heart rate variability (HRV) have become as popular as the many devices available to record the cardiac and autonomic activity (Akselrod et al., 1985). HRV refers to heart beating signal itself as well to the time between consecutive heart beatings. As reported in literature, several diseases and conditions, as myocardial infarction, diabetic neuropathy, and myocardial dysfunction, for example, are related to decreasing HRV (Pagani et al., 1988; La Rovere et al., 1998), pointing to a connection between healthiness and complexity. Also, the effects of aging are known to present higher HRV in younger individuals, compared to elderly ones (Task Force, 1996). The possibility of diagnosis based on non-invasive techniques such as HRV studies is widely investigated (Kurths et al., 1995) and compels us to overcome the difficulties originated on the environmental changes that can affect the signal, eventually pointing out to a false disruption of the dynamics or leading to neglect a real one. Besides environmental conditions, nonstationarities can arise from multiple regulatory mechanisms, for example blood flow and respiration, operating concomitantly and varying over time, with each subsystem presenting its own time scale (Porta et al., 2009), and since the activation of only one control mechanism is not possible, the cardiac record will carry non-stationarities related to the superposition of different regulatory mechanisms (Magagnin et al., 2011). Spectral analysis, for example, relies on the assumption of weak stationarity, where the mean value is constant and the covariance is only dependent on a time shift, but even in controlled environments, it is questionable whether the efficiency of such control ensures these conditions.

The idea of the segmentation applied to time series is to provide patches of the signal where stationarity is verified. BernaolaGalván et al. (2001) introduced a mean based segmentation algorithm which looks for points along the signal where the difference of the mean of the resultant segments is maximal. Since the mean based segmentation has $t$-statistics computed for equal variance samples as the criterion for locating the cutting points, no information about the variances could be inferred. Instead of testing the difference for the mean, we perform a non-parametric segmentation (Camargo et al., 2011), taking into account the whole distribution, with all moments, e.g., mean and variance. In the case of HRV, the known amplitude-frequency coupling of the dominant short-term oscillation, the respiratory sinus-arrhythmia, connects the changes in the variance with the time-dependent covariance, implying non-stationarity (Hirsch and Bishop, 1981). 


\section{MATERIALS AND METHODS}

For analysis, we consider $24 \mathrm{~h}$ measurements of the electrocardiogram of three groups consisting of young and elderly subjects, and patients suffering from congestive heart failure. The groups are composed of 15 patients with congestive heart failure (CHF; 11 males, 4 females, age $56 \pm 11$ years), 18 elderly healthy subjects (EH; 11 males, 7 females, age $50 \pm 7$ years), and 15 young healthy subjects (YH; 11 females, 4 males, age $31 \pm 6$ years). Data for the CHF patients and the $\mathrm{YH}$ subjects are available from PhysioNet (Goldberger et al., 2000). Data for the EH were provided by the Charité in Berlin (Wessel et al., 2003). The series of time intervals between consecutive heart beats, the beat-to-beat intervals, are extracted from the electrocardiograms (Suhrbier et al., 2006). All resultant signals were filtered in order to avoid ectopic beats (Wessel et al., 2007).

We have chosen a non-parametric method based on the comparison of the two segments resulting from a hypothetical cutting, by building the empirical cumulative distribution of the points to the left and to the right of this cut and looking for the cut which would provide the maximal distance between the empirical cumulative distributions, namely the Kolmogorov-Smirnov distance. After determining the position of the cut, we check for its statistical significance, and if necessary, we check for a minimum length. If the required critical distance value, for a given significance level, and the minimum length are verified, then the signal is split into two segments, that on their turns, will be examined in order to find other cutting points. Segmentation ends when no significant difference is found or when the remaining segment is smaller than the minimum. The segmentation procedure can be summarized as follows: given a time series, $\left\{x_{i}, i_{1} \leq i \leq i_{n}\right\}$, a sliding pointer is moved from $i=i_{1}, \ldots, i_{n-1}$ in order to compare the two fragments of sizes $n_{L}=i_{p}-i_{1}+1$, and $n_{R}=$ $i_{n}-i_{p}$, where $i_{p}$ is the position of the pointer. Then one selects the position $i_{\max }$ that maximizes the Kolmogorov-Smirnov (KS) statistics

$$
D \equiv D_{\mathrm{KS}}\left(1 / n_{L}+1 / n_{R}\right)^{-1 / 2},
$$

where $D_{\mathrm{KS}}$ is the maximal distance between the cumulative distributions of the samples. After determining the position $i_{\max }$ of the maximal distance $D, D^{\max }$, one checks statistical significance (at a chosen significance level $\alpha=1-P_{0}$ ) of a potentially relevant cut at $i_{\max }$ by comparison with the result that would be obtained was the sequence random. The critical value is given by the empirical expression

$$
D_{\text {crit }}^{\max }(n)=a(\ln n-b)^{c},
$$

$(a, b, c)=(1.52,1.80,0.14)$ for $P_{0}=0.95, \quad n=n_{L}+n_{R}$. Equation (2) was obtained by determining $D^{\max }$ of a large number of sequences of $n$ independent and identically distributed Gaussian numbers, and then, using the complementary cumulative distribution of $D^{\max }$, we found the critical values for each significance level (Camargo et al., 2011).

The potential cut ticks the first stage if $D^{\max }$ exceeds its critical value for the selected significance level $D_{\text {crit }}^{\max }(n)$. If each resulting segment is greater than a defined minimum $\ell_{0}$, then the pointer is set and the procedure is recursively applied starting from the left patches until no patch is segmented. See Camargo et al. (2011) for further details. We performed the KS-segmentation with $\ell_{0}=30$ and $P_{0}=0.95$. This value of $\ell_{0}$ is chosen in correspondence to the defined higher edge frequency of the very low frequency (VLF) band of heart rate with $0.03 \mathrm{~Hz}$ (Task Force, 1996) providing at least a half period of this frequency in each segment. Figure 1 presents a schematic first step of the segmentation: in (A) we see the original signal, that will be examined point to point, as indicated in (B), splitting the signal into two parts and computing the distance between the empirical cumulative distribution of each side $(\mathbf{C})$. Once this step is completed, one will have a distance $D$ associated to each point of the signal (D) and we look then to the point where $D$ is maximal. We verify the significance of this cut by the comparison with the critical value, and the segment minimum length required, then the signal is cut. As a result, we have the signal split into two segments (E). Repeating the procedure, for each new segment resulting from a cut, until no significant differences found or the length reaches a value smaller than the minimum, one will have the signal fragmented in segments $(\mathbf{F})$.

\section{RESULTS}

By finding stationary segments we are able to analyze the size of these segments and evaluate how the signal changes from one segment to another, looking at the statistical moments given in each patch, for example, mean and variance, in what we can call the intrinsic time scale of the signal (in opposition to arbitrary divisions of the signal). To quantify the non-stationarity, we obtain the length of the segments, $L$, the mean RR interval in each segment, $\mu$, and the variance, $\sigma^{2}$, associated to each segment, as well as the distributed jump size. Figure 2 displays results of the accumulated data from all the subjects in each one of the three groups. In (A) we show the empirical cumulative distribution of the length of the segments $L,(B)$ shows the distributions of the means of each segment, $\mu$, and $(\mathbf{C})$ the distributions of the variances $\sigma^{2}$.

When considering individual distributions of the segment sizes, the empirical cumulative distribution of segment sizes is described by the double exponential curve $A e^{-\left(L-\ell_{0}\right) / L_{1}}+$ $(1-A) e^{-\left(L-\ell_{0}\right) / L_{2}}$. The Mann-Whitney $\mathrm{U}$ test for $L_{2}$ provides significant differences between $\mathrm{CHF}$ and $\mathrm{YH}(p<0.01)$ as well as $\mathrm{CHF}$ and $\mathrm{EH}(p<0.05)$, pointing to differences in the occurrences of large segments between the pathological group and the healthy ones, while the occurrence of short segments shows no significant differences. Comparing the means of the segments does not improve the basic comparison of the means of the complete signal, thus the importance of accessing the second and further moments, if desirable, of each segment. Looking at the distribution of $\sigma^{2}$ of each individual, we were able to fit the curve $\log \left[P D F\left(\sigma^{2}\right)\right]=\alpha\left[\log \left(\sigma^{2}\right)\right]^{2}+\beta\left[\log \left(\sigma^{2}\right)\right]+\gamma$, aiming at individual parameters capable of distinguishing the groups. Performing a Mann-Whitney $U$ test for $\gamma$ values showed in Figure 3 we obtain $p<10^{-4}$ when considering groups $\mathrm{CHF}-\mathrm{YH}$ and $\mathrm{CHF}-\mathrm{EH}$, and $p<0.05$ for $\mathrm{EH}-\mathrm{YH}$. Although significant differences were found between $\mathrm{CHF}$ and the healthy groups when comparing means, the aging effect in the healthy groups is harder to detect, being pointed through symbolic dynamics 

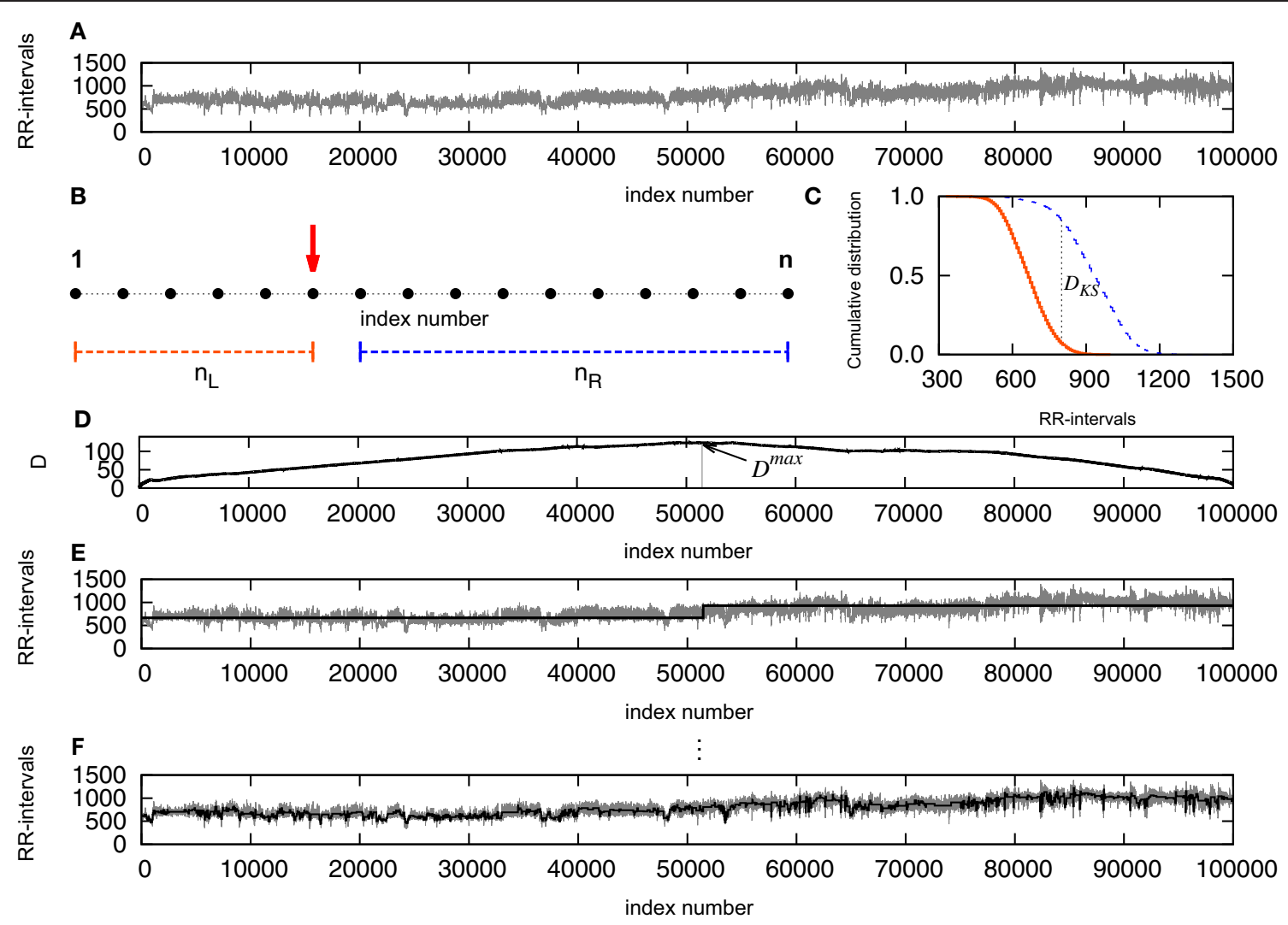

FIGURE 1 | (A) Original signal, that will be examined point to point, as indicated in (B), splitting the signal into two parts and computing the distance between the empirical cumulative distribution of each side (C) Once this step is completed, one will have a distance $D$ associated to each point of the signal (D) and the point where $D$ is maximal is determined. The empirical expression given by Equation (2) is used to verify the significance level of the cut, and if $D$ exceeds $D_{\text {crit }}$ and the segment results not smaller than the minimum, the signal is cut. As a result, the signal is split into two segments (E). Repeating the procedure, for each new segment resulting from a cut, until no significant differences found or the length reaches a value smaller than the minimum, one will have the signal fragmented in segments (F).
POLVAR20 (Wessel et al., 2007) and now through the distribution of variances.

Since it is usual to work with signals subtracted their mean, we compare how the signal subtracted by the segments mean, $R R_{\star}$, can improve the understanding of the non-stationary behavior of the groups. Figure $4 \mathrm{~A}$ shows the trends given by the mean in the segments, $\mu$ for a section of the segmented signal and in (B) we see the signal $R R_{\star}$ resulting from the subtraction of the mean in each segment, $\mu$. Then, we plot in (C) the variance in the detrended signal, $\sigma_{\star}^{2}$, against the variance of $\mu, \sigma_{T}^{2}$, for each subject. The results show correlations between the variability of the trends and the variability of the detrended signals, still displaying higher variability for healthy subjects, yet not providing differentiation due to aging effects.

In order to understand the non-stationary behavior of the groups, we compute the mean variance of the segments $\left\langle\sigma_{(L)}^{2}\right\rangle$ given a segment length, $L$, displayed in Figure 5. One can see that CHF always presents the smaller values of $\left\langle\sigma_{(L)}^{2}\right\rangle$, while $\mathrm{YH}$ and $\mathrm{EH}$ systematically have higher values. It is worth to mention here the similarity of Figure 5 with plots given by detrended fluctuation analysis (DFA) (Peng et al., 1995), where the signal is integrated and then linearly detrended according to fixed sized boxes and then one verifies how the fluctuations behave with varying box sizes. With the segmentation, we have the box sizes replaced by the segments sizes and the fluctuations are given by the mean variances of the segments with given lengths. Computing the scaling exponents, obtained through the numerical fittings indicated in Figure 5 by solid lines, we obtain the slopes $\xi_{\mathrm{CHF}}=0.6, \xi_{\mathrm{EH}}=0.4$, and $\xi_{\mathrm{YH}}=0.2$. Results from DFA have shown that, in the range of $10^{2} \sim 10^{4}$ beats, the scaling exponent of the pathological group approaches to 1.5, corresponding to Brownian noise, that is integrated white noise. For random walk, DFA provides a scaling exponent of 0.5 . In our segmentation analysis, as we do not integrate the signal, the correspondent pathological scaling exponent $\xi_{\mathrm{CHF}}=0.6$ indicates that random walk fluctuations dominate the dynamics of this group, in good accordance with DFA interpretation. For the $\mathrm{EH}$ and $\mathrm{YH}$ groups, the exponents $\xi_{\mathrm{EH}}=0.4$, and $\xi_{\mathrm{YH}}=0.2$ indicate power law correlations associated to the interchange of large and small $\mathrm{RR}$ intervals. In this sense, we can confirm the loss of complexity due to pathological condition and aging.

To the extent of standard HRV analysis, in Figure 6 we plot the number of segments with $L>300, L_{>300}$, corresponding to approximately $5 \mathrm{~min}$, the shortest required segment length of 


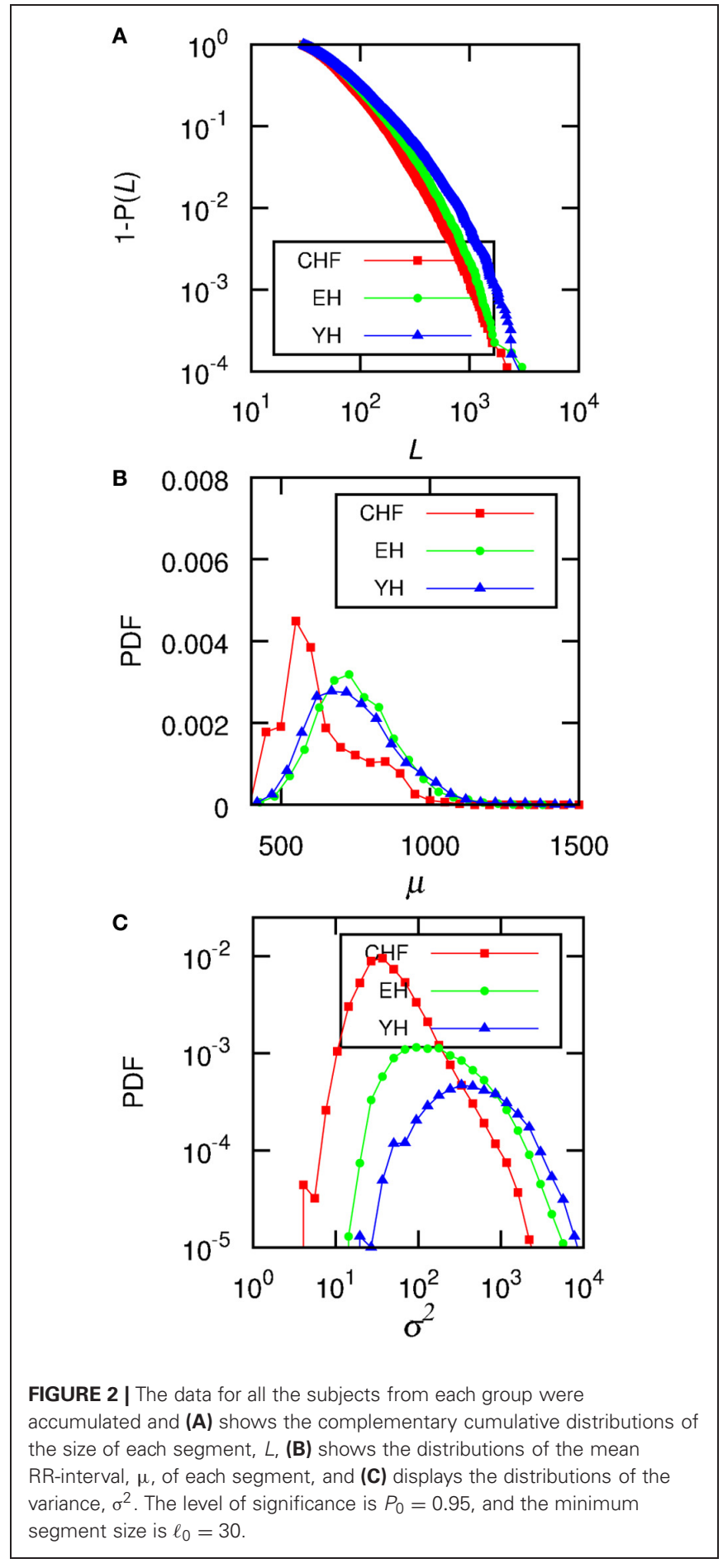

HRV analysis (Task Force, 1996) and $\% \mu_{50}$, the percentage of differences of the mean of two consecutive segments $\left|\mu_{i+1}-\mu_{i}\right|>$ $50 \mathrm{~ms}$ in an analogy to the standard HRV measure pNN50, the percentage of consecutive RR intervals differing by more than $50 \mathrm{~ms}$ (Task Force, 1996). Figure 6 reveals that the pathological group, $\mathrm{CHF}$, presents smaller $\% \mu_{50}$ than the healthy groups $\mathrm{EH}$ and $\mathrm{YH}$, with the last exhibiting also higher number of long segments. The aging affects the occurrence of larger segments as we

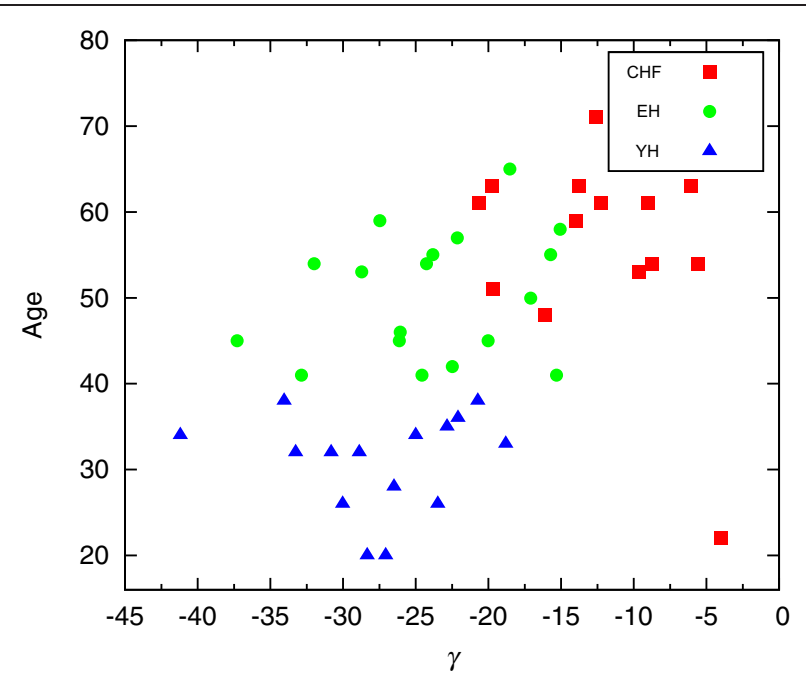

FIGURE 3 | Scatter plot of the parameter giving the best fit of $\log \left[P D F\left(\sigma^{2}\right)\right]=\alpha\left[\log \left(\sigma^{2}\right)\right]^{2}+\beta\left[\log \left(\sigma^{2}\right)\right]+\gamma$. Performing a Mann-Whitney $U$ test for $\gamma$ a $p$-value smaller than $10^{-4}$ is obtained when considering groups $\mathrm{CHF}-\mathrm{YH}$ and $\mathrm{CHF}-\mathrm{EH}$, and $p<0.05$ for $\mathrm{EH}-\mathrm{YH}$.

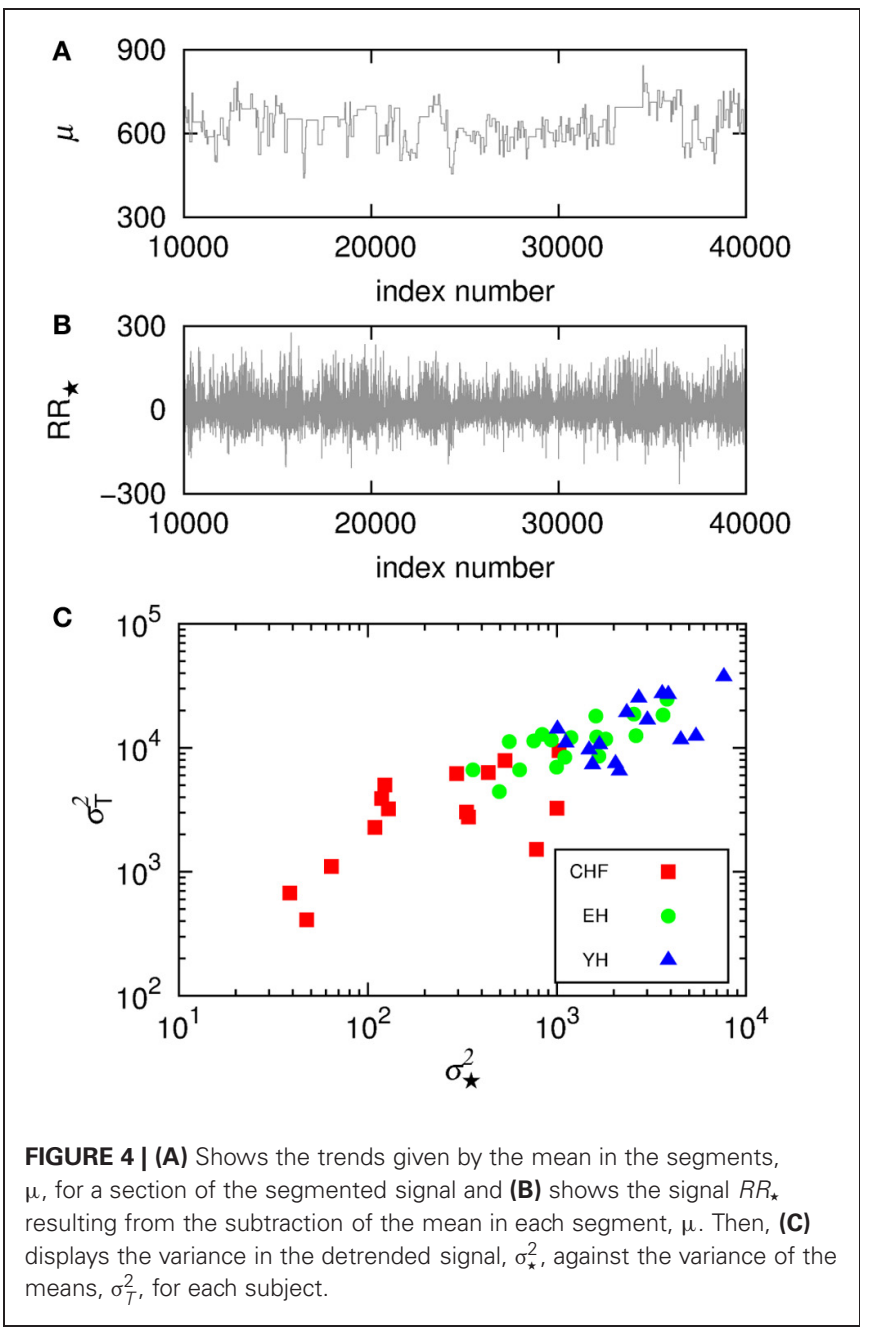




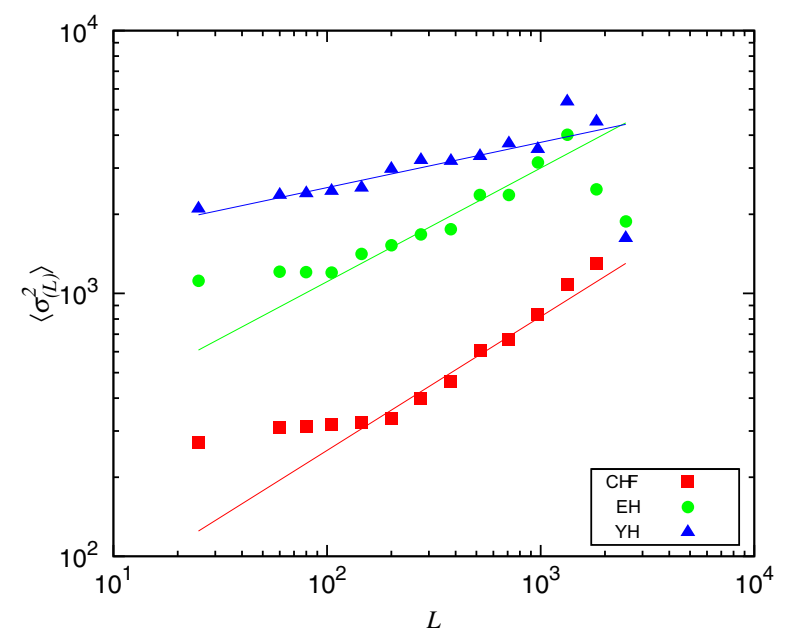

FIGURE 5 | Mean variance of the segments $\left\langle\sigma_{L}^{2}\right\rangle$ given the segments length, $L$. The scaling exponents were obtained through numerical fittings, indicated by solid lines, with slopes $\xi_{\mathrm{CHF}}=0.6, \xi_{\mathrm{EH}}=0.4$, and $\xi_{\mathrm{YH}}=0.2$.

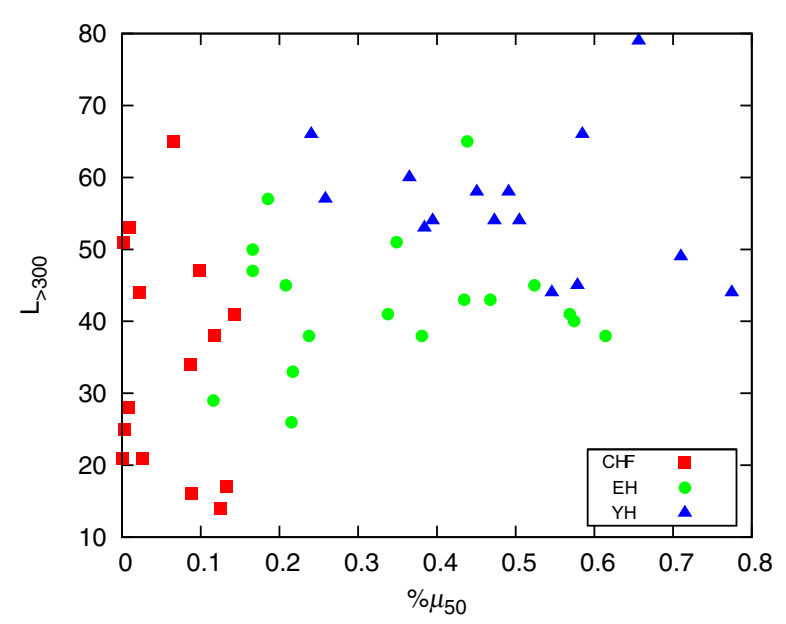

FIGURE 6 | Plot of the number of segments with $L>300$ (corresponding to approximately $5 \mathrm{~min}$ ) and $\% \mu_{50}$, the percentage of differences of the mean of two consecutive segments $\left|\mu_{i+1}-\mu_{i}\right|>50 \mathrm{~ms}$.

know that the oldest subjects of $\mathrm{EH}$ are clustered around $L_{>300}=$ 40 and the oldest patients of CHF group are clustered around $L_{>300}=30$. We found high positive correlations between $\% \mu_{50}$ and standard deviation of the signal, $s d N N$, as well as a negative correlation between $L_{>300}$ and the normalized very low frequency power, $V L F / P$, linking the segmentation outcomes to standard measures. The first one shows that $s d N N$ is dominated by the large jumps whereas the second correlation reflects the reduction of very low frequencies for longer segments. For the pathological group, CHF, the correlation between $s d N N$ and $\% \mu_{50}$ is 0.94 , while healthy groups, $\mathrm{YH}$ and $\mathrm{EH}$, present a correlation of 0.90 . For $V L F / P$ and $L_{>300}$ we have a correlation of -0.77 for $\mathrm{CHF}$ and -0.81 for $\mathrm{YH}$ and $\mathrm{EH}$.

\section{DISCUSSION}

In this paper we present the analysis of non-stationarities in heart rate by means of a non-parametric segmentation algorithm, being able to detect significant differences between $\mathrm{CHF}$ and age-matched $\mathrm{EH}$, as well as $\mathrm{CHF}$ and $\mathrm{YH}$. Also, significant differences between $\mathrm{YH}$ and $\mathrm{EH}$ can be detected, showing the aging effect in the loss of complexity of the heart rate. We found a correspondence between DFA results, suggesting that random walk fluctuations dominate the dynamics of the pathological group. For the healthy groups, $\mathrm{EH}$ and $\mathrm{YH}$, the long term correlations in the interchange of large and small RR intervals appears to be larger for $\mathrm{EH}$ and smaller for $\mathrm{YH}$, denoting diminished non-stationarities due also to aging and pathological condition. Although KS-segmentation is limited to long term observations, since short data provide poor statistics of the segments, when considering previous segmentation methods, this choice for using the Kolmogorov-Smirnov distance instead of the mean of the segments provides the possibility of characterizing all the statistical moments of the segments, particularly the changes in the variance. According to our results, comparing means or standard deviation is not efficient to distinguish $\mathrm{EH}$ and $\mathrm{YH}$, but the aging effect can be indicated by the variance in the segments. The aging effect has been detected when applying POLVAR20 (Wessel et al., 2003), which gives a good classification of the groups; however, no information about the dynamics underlying the systems is provided. Additionally, this segmentation procedure differs from arbitrarily splitting the signal in equal size segments and testing whether they come from the same distribution or not, rather it is the test for significant differences that determines the lengths of segments.

In terms of standard HRV analysis, a high positive correlation between $\% \mu_{50}$ and $s d N N$ shows that the standard deviation is dominated by the large jumps. A negative correlation between $L_{>300}$ and $V L F / P$ reflects the reduction of very low frequencies for longer segments. Periodic breathing is prevalent in $30-50 \%$ of the patients with CHF (Hall et al., 1996; Lorenzi-Filho et al., 2005), and it has been reported (Lorenzi-Filho et al., 1999) that periodic breathing leads to modulation of the RR intervals in the VLF band, what would cause the algorithm to detect a larger number of short segments for CHF.

Through the outcomes of segmentation we have access to time characteristics of the signal that were no longer available, making possible a different approach to quantify non-stationarities in HRV analysis. Results are in agreement with previous knowledge and do not require arbitrary thresholds or exclude fragments of the time series. The individual risk stratification ability of this method relies on further applications to cardiological data bases.

\section{ACKNOWLEDGMENTS}

We acknowledge Prof. Schirdewan for providing data. Sabrina Camargo and Celia Anteneodo acknowledge CNPq (Brazilian agency) for financial support. Maik Riedl, Niels Wessel, and Jürgen Kurths acknowledge the Deutsche Forschungsgemeinschaft, grant numbers DFG RI2016/2-1, WE 2834/5-1, KU837/29-2, and KU837/35-1. 


\section{REFERENCES}

Akselrod, S., Gordon, D., Madwed, J. B., Snidman, N. C., Shannon, D. C., and Cohen, R. J. (1985). Hemodynamic regulation: investigation by spectral analysis. Am. J. Physiol. Heart Circ. Physiol. 249, H867-H875.

Bernaola-Galván, P., Ivanov, P. C., Amaral, L. A. N., and Stanley, H. E. (2001). Scale invariance in the nonstationary of human heart rate. Phys. Rev. Lett. 87:168105. doi: 10.1103/PhysRevLett.87.168105

Camargo, S., Queirós, S. M. D., and Anteneodo, C. (2011). Nonparametric segmentation of nonstationary time series. Phys. Rev. E 84:046702. doi: 10.1103/PhysRevE.84.046702

Goldberger, A. L., Amaral, L. A. N., Glass, L., Hausdorff, J. M., Ivanov, P. C., Mark, R. G., et al. (2000). Physiobank, physiotoolkit, and physionet:components of a new research resource for complex physiologic signals. Circulation 101, e215-e220. Circulation Electronic Pages: http://circ.ahajournals.org/ cgi/content/full/101/23/e215.

Hall, M. J., Xie, A., Rutherford, R., Ando, S., Floras, J. S., and Bradley T. D. (1996). Cycle length of periodic breathing in patients with and without heart failure. Am. J. Resp. Crit. Care Med. $154,376$.

Hirsch, J., and Bishop, B. (1981). Respiratory sinus arrhythmia in humans: how breathing pattern modulates heart rate. Am. J. Physiol. Heart Circ. Physiol. 241, H620-H629.

Kurths, J., Voss, A., Saparin, P., Witt, A., Kleiner, H. J., and Wessel, N. (1995). Quantitative analysis of heart rate variability. Chaos 5, 88-94.

La Rovere, M. T., Bigger, J. T. Jr., Marcus, F. I., Mortara, A., and Schwartz, P. J. (1998). Baroreflex sensitivity and heart-rate variability in prediction of total cardiac mortality after myocardial infarction. atrami (autonomic tone and reflexes after myocardial infarction) investigators. Lancet 351, 478-484.

Lorenzi-Filho, G., Dajani, H. R., Leung, R. S., Floras, J. S., and Bradley, T. D. (1999). Entrainment of blood pressure and heart rate oscillations by periodic breathing. Am. J. Resp. Cri. Care Med. 159, 1147-1154.

Lorenzi-Filho, G., Genta, P. R., Figueiredo, A. C., and Inoue, D. (2005). Cheyne-stokes respiration in patients with congestive heart failure: causes and consequences. Clinics 60, 333.

Magagnin, V., Bassani, T., Bari, V., Turiel, M., Maestri, R., Pinna, G. D., et al. (2011). Nonstationarities significantly distort short-term spectral, symbolic and entropy heart rate variability indices. Physiol. Meas. 32, 1775.
Pagani, M., Malfatto, G., Pierini, S., Casati, R., Masu, A. M., Poli, M., et al. (1988). Spectral analysis of heart rate variability in the assessment of autonomic diabetic neuropathy. J. Auton. Nerv. Syst. 23, 143-153.

Peng, C.-K., Havlin, S., Stanley, H. E., and Goldberger, A. L. (1995). Quantification of scaling exponents and crossover phenomena in nonstationary heartbeat time series. Chaos 5, 82 .

Porta, A., Di Rienzo, M., Wessel, N., and Kurths, J. (2009). Addressing the complexity of cardiovascular regulation. Phil. Trans. R. Soc. A 367, 1215.

Suhrbier, A., Heringer, R., Walther, T., Malberg, H., and Wessel, N. (2006). Comparison of three methods for beat-to-beat-interval extraction from continuous blood pressure and electrocardiogram with respect to heart rate variability analysis. Biomed. Tech. 51, 70.

Task Force of the European Society of Cardiology and the North American Society of Cardiology. (1996). Heart rate varibility. Standards of measurement, physiological interpretation, and clinical Use. Eur. Heart J. 17, 354 .

Wessel, N., Malberg, H. Bauernschmitt, R., and Kurths, J. (2007). Nonlinear methods of cardiovascular physics and their clinical applicability. Int. J. Bifurcation Chaos 17, 3325.
Wessel, N., Schirdewan, A., and Kurths, J. (2003). Intermittently decreased beat-to-beat variability in congestive heart failure. Phys. Rev. Lett. 91:119801. doi: 10.1103/PhysRevLett.91.119801

Conflict of Interest Statement: The authors declare that the research was conducted in the absence of any commercial or financial relationships that could be construed as a potential conflict of interest.

Received: 28 March 2013; accepted: 26 April 2013; published online: 15 May 2013

Citation: Camargo S, Riedl $M$, Anteneodo C, Kurths $J$ and Wessel $N$ (2013) Diminished heart beat nonstationarities in congestive heart failure. Front. Physiol. 4:107. doi: 10.3389/fphys. 2013.00107

This article was submitted to Frontiers in Computational Physiology and Medicine, a specialty of Frontiers in Physiology.

Copyright (C) 2013 Camargo, Riedl, Anteneodo, Kurths and Wessel. This is an open-access article distributed under the terms of the Creative Commons Attribution License, which permits use, distribution and reproduction in other forums, provided the original authors and source are credited and subject to any copyright notices concerning any thirdparty graphics etc. 